\title{
Unsteady Fluid-Structure-Jet Interaction Modeling Methods for Agile High-Speed Vehicles
}

\author{
Ryan C. Kitson* and Carlos E. S. Cesnik ${ }^{\dagger}$ \\ University of Michigan, Ann Arbor, MI, 48109, USA
}

\begin{abstract}
This paper considers the nonlinear and unsteady loads environment of an agile highspeed vehicle with reaction control jets, a large flight envelope, and structural degrees of freedom to account for the flexibility of the slender vehicle. Steady-state and unsteady CFD solutions of the fluid-structure-jet interaction are calculated throughout the flight envelope to build a reduced order model. Two surrogate modeling methods are compared to assess the applicability to the fluid-structure-jet interaction and wide variation in flight conditions: a CFD-based steady-state Kriging approximation and linear convolution with a nonlinear correction. The surrogate models are then compared to FUN3D CFD solutions to verify their accuracy. Overall, this work bridges the gap between system identification at single flow conditions and flight simulations of an unknown trajectory.
\end{abstract}

\section{Nomenclature}

A Matrix of surface pressures from the CFD solution

$A_{\text {ref }} \quad$ Reference area, $\mathrm{m}^{2}$

AoA Angle of Attack, deg

$A o S \quad$ Sideslip, deg

$C_{x} \quad$ Axial force coefficient

$C_{y} \quad$ Side force coefficient

$C_{z} \quad$ Normal force coefficient

$C_{m} \quad$ Moment coefficient

$F \quad$ Fluid parameters (Mach, Altitude, Angle of Attack, Angle of Sideslip)

$H \quad$ Step response

$H_{i}^{j} \quad$ Step response corresponding to load $j$ and input $i$

$J \quad$ Jet parameters (total pressure of the attitude and divert jets)

$L_{r e f} \quad$ Reference length, $\mathrm{m}$

$S \quad$ Structural parameters (longitudinal and lateral first bending mode amplitude)

$U \quad$ Left singular vectors of $A$

$V \quad$ Right singular vectors of $A$

$k \quad$ Step response time index, $1 \ldots n$

$p \quad$ Pressure, $\mathrm{Pa}$

$q \quad$ Dynamic pressure, $\mathrm{Pa}$

$u \quad$ Model input

$u^{i} \quad$ Model input corresponding to input $i$

$x, y, z$ Body-fixed axes with $x$ positive aft, $y$ positive right, $z$ positive up

$y \quad$ Model output

$y^{j} \quad$ Linearized model output corresponding to load $j$

$y_{\text {linear }}^{s}$ Linearized model steady-state output

$y_{\text {nonlinear }}^{s}$ Nonlinear steady-state model output

$y_{\text {linear }}^{u}$ Linearized model output

*Ph.D. Candidate, Department of Aerospace Engineering, kitson@umich.edu; AIAA Student Member

${ }^{\dagger}$ Professor, Department of Aerospace Engineering, cesnik@umich.edu; AIAA Fellow 
$y_{\text {nonlinear }}^{u}$ Unsteady model output including nonlinear correction factor

$\Sigma \quad$ Singular values of $A$

$\delta_{1} \quad$ Attitude jet total pressure, $\%$ maximum

$\delta_{2} \quad$ Starboard divert jet total pressure, $\%$ maximum

$\delta_{3} \quad$ Port divert jet total pressure, $\%$ maximum

$\eta_{1} \quad$ Lateral first bending mode deformation, $\%$ tip deflection

$\eta_{2} \quad$ Longitudinal first bending mode deformation, \% tip deflection

Subscripts

$i, j \quad$ Row and column indexes corresponding to CFD surface mesh point and sample conditions

\section{Introduction}

Conventional air vehicles have a standard mission that often leads to defining a "cruise" phase and a "trim" condition. However, maneuverable and agile vehicles that maneuver to reach a moving target do not have a pre-defined trajectory and may not have a clearly defined reference flight condition. The agile vehicle maneuvers may be very aggressive and may operate at the extremes of flow conditions. Quality models and methods for calculating the unsteady loads on the vehicle are required for accurate analysis of the vehicle response during flight simulations. However, the model must capture the wide range of flow conditions such as very high angles of attack over the range of flight Mach number and altitude.

High-speed maneuverable vehicles for air-to-air missions are slender with large aerodynamic and inertial loading that may lead to significant structural deformation. In addition, performance can be increased using an alternative control system such as attitude control jets instead of traditional control surfaces. However, these two effects introduce a fluid-structure interaction and a jet interaction to the vehicle analysis. In addition, it is not well known how the structural deformation will affect the jet interaction or vice versa. Therefore, the analysis of a high-performance maneuverable vehicle requires methods that account for the coupled fluid-structure-jet interaction.

Previous experimental studies have been conducted regarding the jet interaction with a supersonic crossflow and the characteristic steady-state flow condition has been well documented. Spaid and Zukoski, ${ }^{1}$ Spaid and Cassel, ${ }^{2}$ and Zukoski and Spaid ${ }^{3}$ experimentally identified the flow structure and developed preliminary models for the interaction. Roger ${ }^{4}$ summarized the jet interaction literature of the previous 50 years and identified the common trends, critical parameters, and highlighted the remaining challenges. One of the challenges was that the community has taken a sporadic approach to the problem and there is a lack of a common methodology that could be used for multiple vehicles, flight conditions, and jet conditions.

Various empirical and theoretical modeling efforts have been made to correlate the flow and jet conditions to the flow velocity or surface quantities. ${ }^{5-8}$ However, these are primarily focused on the flat plate results and typically the longitudinal jet plane, which neglects the three-dimensional effects. Broadwell ${ }^{9}$ related the jet interaction problem to the blast wave equations to approximate the shock boundary and pressure profile in the lateral directions, but this was also focused on a flat plate and is not expected to hold for a complex geometry. Computational modeling using the Reynolds-averaged Navier-Stokes equations has been done and demonstrates the ability to analyze the jet interaction problem for various configurations, flow and jet conditions. ${ }^{10-18}$ In addition, Sahu et al. ${ }^{12}$ has coupled the rigid-body equations of motion for a projectile to the fluid dynamics equations for a coupled flight simulation of a rigid vehicle with jet interaction. Computational modeling has introduced some additional challenges such as turbulence modeling ${ }^{16}$ and highlighted important phenomena such as the jet transient forces. ${ }^{17,19,20}$ Considering a maneuverable vehicle in a dynamic flight environment, it is critical to understand the dynamics of the jet interaction with varying flow and jet conditions. Further detailed analysis using the large eddy simulation (LES) approach has been done as well ${ }^{21,22}$ to better understand the vortex shedding, flow mixing, and turbulent boundary layer interaction with the jet. Computational analysis continues to further the understanding of the jet interaction problem, but the computational cost increases and robustness decreases as the models and methods become refined or higher-order.

Reduced order modeling of the jet interaction and of the overall aerodynamic loading can maintain a level of accuracy and greatly reduce the computational cost. VanderWyst et al. ${ }^{23,24}$ developed a CFD-based surrogate of the loading for a high-speed vehicle with jet interaction that demonstrate multiple approaches to the high-dimensional problem. Moreover, there is a significant amount of previous work regarding unsteady loads modeling that can be applied to the current fluid-structure-jet interaction problem of a slender 
high-speed vehicle. One approach is to use linear convolution with the vehicle step or impulse response to approximate the loads with an arbitrary input during flight simulations. ${ }^{25,26}$ The linear convolution technique breaks down as the arbitrary input becomes significantly larger than the training step input, which is why Skujins and Cesnik $^{26}$ implemented a nonlinear correction factor based on nonlinear steady-state analysis $a$ priori. The nonlinear correction factor proved to be quite accurate over multiple Mach regimes and may be a successful approach to modeling the nonlinear jet interaction over a wide range of flow and jet conditions. Another approach is to use an autoregressive moving-average (ARMA) model that is fit to a characteristic vehicle response then used to approximate the vehicle loading with an arbitrary input. ${ }^{27}$ The number of retained coefficients in the ARMA model determine the relative gains in memory storage and loss of information relative to the convolution approach. An unsteady loads model of the coupled fluid-structure-jet interaction problem should be developed that captures the nonlinear effects such as control reversal and the jet transient loads in a dynamic flight environment.

The fluid-structure-jet interaction, which is the addition of structural degrees of freedom to the jet interaction problem, is not well understood. The bulk of jet interaction research is not concerned with the deformation of the surface on which the jet is mounted. However, this problem is critical not only for a freeflight vehicle, but a flexible vehicle as well. The change in rigid-body velocities and structural deformation will change the flow conditions and, therefore, the forces and moments applied by the jet interaction with the flow.

Agile vehicles have a large flight envelope that complicates the modeling strategy. Vehicles performing aggressive maneuvers do not have a predefined trajectory, which prevents any significant down-selection of control input and vehicle state variable combinations during model development. In addition, the vehicle is likely to experience a highly dynamic flight environment with large angles of inclination to the flow, large angular rates, flow disturbances from the control system and vehicle deformation. These factors will result in complex phenomena such as flow separation and shock-boundary layer interaction that are common and critical to accurate loads calculation for agile high-speed vehicles

This work investigates methods to modeling the unsteady loads due to fluid-structure-jet interaction of a agile high-speed vehicle. There is an emphasis on the extreme flow conditions and the wide range of the flight envelope of the nonlinear system. A representative slender vehicle is used as the test case throughout and the NASA FUN3D CFD $\operatorname{code}^{28}$ is used to calculate the surface load distribution. The unsteady prediction methods are well known for analysis at a single flight condition. However, the vehicle is expected to operate in a very large flight envelope and has many degrees of freedom. In addition, the nonlinearity of the aerodynamics and jet interaction further complicates the problem. Therefore, a method of adapting these modeling approaches to the wide range of flight conditions is developed. The final model is included in a coupled aeroelastic and flight mechanics framework to analyze the vehicle performing aggressive maneuvers in free flight.

\section{Numerical Methods}

This section describes the approach to developing a reduced model of the unsteady surface loads due to fluid-structure-jet interaction (FSJI) of a high-speed vehicle. Emphasis is placed on the ability to model and approximate FSJI over a range of altitudes and Mach numbers.

First, the steady-state solution is calculated over the whole range of flight conditions, flow angles, structural deformation, and jet conditions. This provides a set of solutions that can be compressed to find the basis vector that describe the surface pressure. The coefficients to these basis vectors can then be correlated with the flight conditions, vehicle velocities, deformation, and jet conditions using a kriging-based approximation technique.

Next, the step response to a change in vehicle orientation, deformation, and jet conditions is calculated for a range of flow conditions. The step change is chosen to be large enough to excite as much of the system as possible. The step responses of the vehicle are then used to approximate the unsteady loads with an arbitrary input history. The first uses a steady-stead CFD surrogate model to predict the applied forces. The second method uses the step response and linear convolution with the input history to approximate the surface loads. These approaches use the same CFD solutions as training data and have been previously used to predict the unsteady aerodynamic loads of flight vehicles. ${ }^{25,26}$ 


\section{A. Steady-state Fluid-Structure-Jet Modeling}

The vehicle surface pressure is described in terms of orthogonal vectors and the predicted coefficients of each vector are then used to reconstruct the surface pressure due to jet interaction. The kriging inputs are the $F$, $S$, and $J$ parameters corresponding to the atmospheric, flow, structure, and jet conditions. The outputs are the $m$ coefficients of the orthogonal vectors in the surface mesh $N$-dimensional space, where $N$ is number of cells in the surface mesh and $m$ is the number of orthogonal vectors. The singular value decomposition (SVD) is used to calculate the orthogonal vectors. First, a matrix $A$ is created with each column vector representing a particular CFD solution,

$$
A=p_{C F D}\left(x_{i}, y_{i}, z_{i}, F_{j}, S_{j}, J_{j}\right)
$$

The SVD of this matrix yields the orthogonal basis $U$, singular values $\Sigma$ and orthogonal basis $V$ where $A V=U \Sigma$. The singular values represent the energy associated with each vector and all vectors are retained in this work. This process is related to the proper orthogonal decomposition of the matrix $A$ where the matrix of basis vectors is equal to the retained vectors of $U \Sigma$ and the coefficients are equal to the retained rows of $V$. Each row of $V$ represents the coefficients at each angle of attack and pressure ratio condition. Therefore, if the coefficients of $V$ can be predicted at alternate input conditions as $\tilde{V}$, an approximation of the surface pressure is $\tilde{A}=U \Sigma \tilde{V}^{T}$ ?

\section{B. Linear Convolution and Nonlinear Correction Factor}

The linear convolution method uses the step response with the arbitrary input history and convolves the two functions of time to approximate the solution. For a linear system, this would be the exact solution. This method is based on previous work by Raveh and Mavris ${ }^{25}$ that showed how a step response as opposed to an impulse response could be used to calculate unsteady loads. For example, during the vehicle simulation the angle of attack history $u$ is used with the step response $H$ with the discrete version of Duhamel's integral to calculate the aerodynamic load at the current time step $y[n]$ as

$$
y[n]=u[0] H[n]+\sum_{k=1}^{n}(u[k]-u[k-1]) H[n-k]
$$

The first term $u[0] H[n]$ represents the steady aerodynamic load at index 0 while the second term is the unsteady component due to an arbitrary input from index 1 to $n$. Therefore, the steady term can come from a more accurate source such as a steady-state CFD solution and the unsteady contribution of the most recent time steps can be calculated using convolution. The result is a linear approximation of the CFD solution for the arbitrary input.

For a linear system and for small inputs this approach works well, but it does not hold as the input becomes large for a nonlinear system. Therefore, this work implements a nonlinear correction factor that is applied to the linear approximation and is based on the steady-state model that has been developed. Skujins and Cesnik $^{26}$ used this approach and it is based on the assumption that the ratio of the unsteady nonlinear solution to the unsteady linear solution is equal to the ratio of the nonlinear steady-state solution and the linear steady-state solution, i.e,

$$
\left(\frac{y_{\text {nonlinear }}}{y_{\text {linear }}}\right)^{u}=\left(\frac{y_{\text {nonlinear }}}{y_{\text {linear }}}\right)^{s}
$$

The term $y_{\text {linear }}^{s}$ is calculated using the property of superposition for linear systems and is the sum of each response for the given combination of inputs. The steady CFD solutions are represented by the $y_{\text {nonlinear }}^{s}$ term. At each time step within a flight simulation the term $y_{\text {linear }}^{u}$ is calculated using linear convolution of all the inputs and summed. $y_{\text {nonlinear }}^{u}$ is the final approximation of the nonlinear unsteady output.

A step response for each input is recorded at each sample flight condition and then decomposed into the dominant responses using the singular value decomposition. Therefore, at a new flight condition the amplitude of these modes are approximated to recreate the step responses an use linear convolution to find the linear response. Finally, the nonlinear correction factor is applied to calculate the total response.

\section{Numerical Approach}

This section begins with defining a vehicle model in terms of geometry, structural properties, and control jet properties. Next, the steady-state load distribution over the vehicle surface is calculated for various 
combinations of fluid, structure, and jet conditions. The steady-state surrogate model can be used for steady and quasi-steady analysis, but is not sufficient for capturing unsteady effects. The dynamic response of the vehicle to changes in the fluid, structure, and jet parameters is calculated to create a linearized unsteady model of the problem. This process is repeated for multiple flight conditions within the flight envelope and interpolated to approximate the linearized model at unsampled flight conditions. To account for nonlinear effects the steady-state surrogate is used as a correction to the linearized approximation during flight simulation.

\section{A. Vehicle Model}

The vehicle used for this study was developed in Kitson and Cesnik ${ }^{29}$ for flight simulation studies of a flexible high-speed vehicle. The basic dimensions and flight conditions for the vehicle are shown in Table 1 and are inspired by existing vehicles, such as the Sidewinder and AMRAAM. The outer mold line of the model, shown in Figure 1, was chosen to move the center of pressure aft of the center of gravity based on the lack of fin surfaces that are conventionally located at the tail end of the vehicle. Markers for the center of pressure (C.P.) and center of gravity (C.G.) have been added to the figure based on the parameters of the vehicle. Conventional fin surfaces are not included in this model due to their loss of effectiveness at high angles of attack. Instead, reaction control jets are located at $25 \%$ of the vehicle length normal to the body surface to control the vehicle orientation. The baseline configuration in the study has a uniform mass and stiffness distribution, therefore the low-frequency free-free vibration mode shapes are similar to a uniform free-free beam shown in Figure 2.

Table 1. Basic properties and flight conditions for the vehicle

\begin{tabular}{lrl}
\hline \hline Property & Value & Unit \\
\hline Total mass (uniform distribution) & 85 & $\mathrm{~kg}$ \\
Length, $L_{r e f}$ & 3 & $\mathrm{~m}$ \\
Reference Area, $A_{r e f}$ & 0.195 & $\mathrm{~m}^{2}$ \\
Diameter at mid-length & 0.13 & $\mathrm{~m}$ \\
Mach & $2-4$ & \\
Altitude & $12-30$ & $\mathrm{~km}$ \\
\hline
\end{tabular}

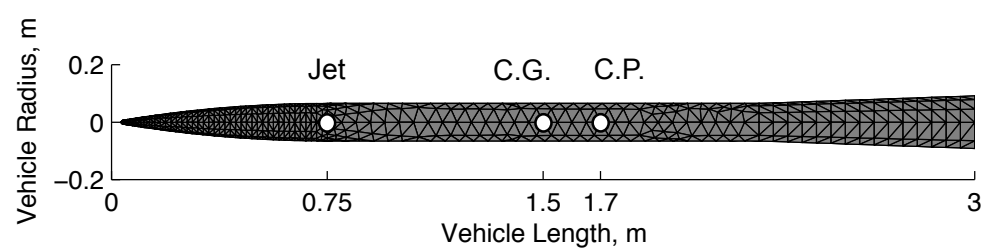

Figure 1. Side view of the undeformed axisymmetric vehicle ${ }^{29}$

\section{B. Steady-State FSJI Modeling}

A steady-state model is developed in this work to account for nonlinear effects since the unsteady modeling approach is based on a linearized approach. The steady-state model approximates the load distribution to calculate rigid-body and structural forces on the vehicle at a combination of fluid, structure, and jet conditions within the flight envelope. This section details the steady-state modeling development from initial sampling to cross-validation.

The surrogate model is based on several samples of the CFD steady-state solution of the vehicle. LatinHypercube Sampling was used to calculate the sample coordinates within the 9 dimensions for the model input. This approach was selected to develop a pseudo-random sampling of the input domain as opposed to a uniform grid sampling. In addition, the parameters were processed to produce a sample set that creates 

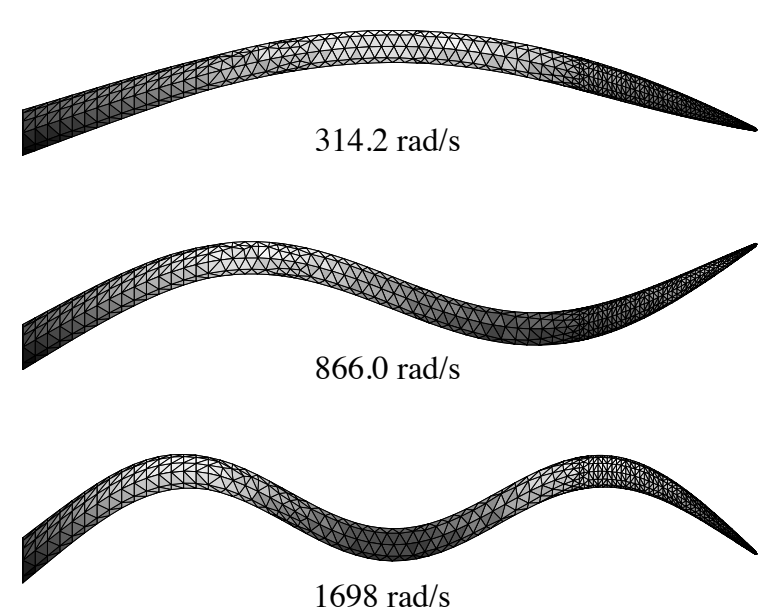

Figure 2. Free-free vibration mode shapes and corresponding frequencies of the vehicle with uniform mass and stiffness ${ }^{29}$

clusters near the mean condition expected for the vehicle. Table 2 lists the 9 pre-processed inputs and the final inputs for the model. Figure 3 shows the distribution of the final inputs that features the clusters near the origin of the flow, structure, and jet parameters. The flow and structure input parameters were clustered using a polar coordinate system based on the total value (e.g. total deformation) and an angle to relate two of the dimensions in the final surrogate model. The jet parameters were related using an artificial spherical coordinate system. The jet throttle is the coordinate from 0-1 that corresponds to the minimum and maximum jet total pressure. A negative jet throttle value corresponds to the jet located on the opposite jet in the body z-coordinate direction. The three reference jets attitude, divert 1 , divert 2 are the jets on the positive-z vehicle surface that correspond to positive y-rotation, negative $\mathrm{x}$-rotation, positive $\mathrm{x}$-rotation using the body-fixed coordinate system with $\mathrm{x}$-axis out the nose, $\mathrm{y}$-axis right, $\mathrm{z}$-axis forming a right-handed system.

Table 2. Input parameters used with Latin-Hypercube Sampling and the surrogate model inputs

\begin{tabular}{cc}
\hline \hline Latin Hypercube Sampling Input & Steady-State Surrogate Model Input \\
\hline Altitude & Altitude \\
Mach & Mach \\
Total Angle of Incidence & Angle of Attack \\
Roll Angle & Angle of Sideslip \\
Total Deformation & Longitudinal First Bending Mode Normalized Tip Displacement \\
Deformation Body-Frame Angle & Lateral First Bending Mode Normalized Tip Displacement \\
Jet Throttle & Attitude Jet Throttle \\
Jet $\theta$ & Divert Jet 1 Throttle \\
Jet $\phi$ & Divert Jet 2 Throttle \\
\hline
\end{tabular}

\section{Unsteady FSJI Modeling}

The unsteady component of the vehicle loads is required to accurately represent the dynamic flow, structure and jet conditions. These unsteady effects vary depending on the flight conditions, i.e. changes in altitude and Mach number, which must be accounted for in the surrogate model development. The linear convolution of step or impulse responses has been used in the past ${ }^{25,26}$ to approximate the response at un-sampled flight conditions. This work examines the applicability of the convolution approach used by Skukins and Cesnik ${ }^{26}$ to the unsteady FSJI problem over its flight envelope.

In this study the inputs are parameters from the fluid, structure, and jet domains listed in Table 3. For each input, a step change equivalent to $1 \%$ of the max amplitude of the input parameter is applied and the 


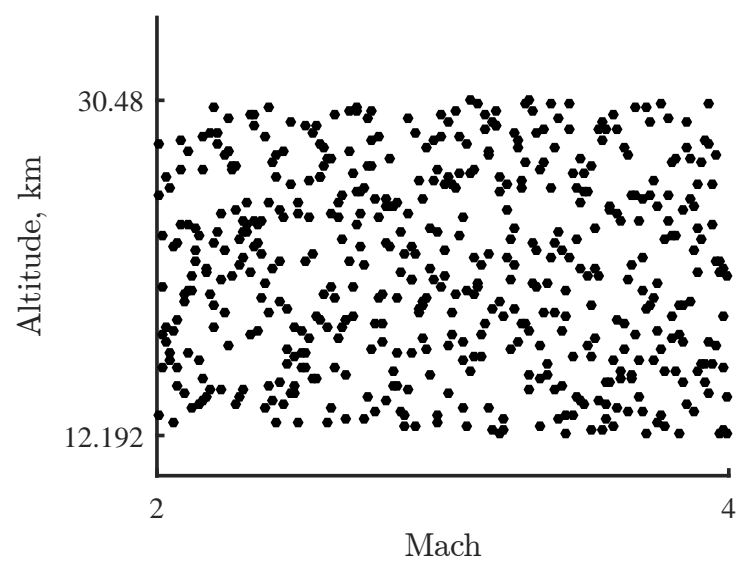

(a) Flight Conditions

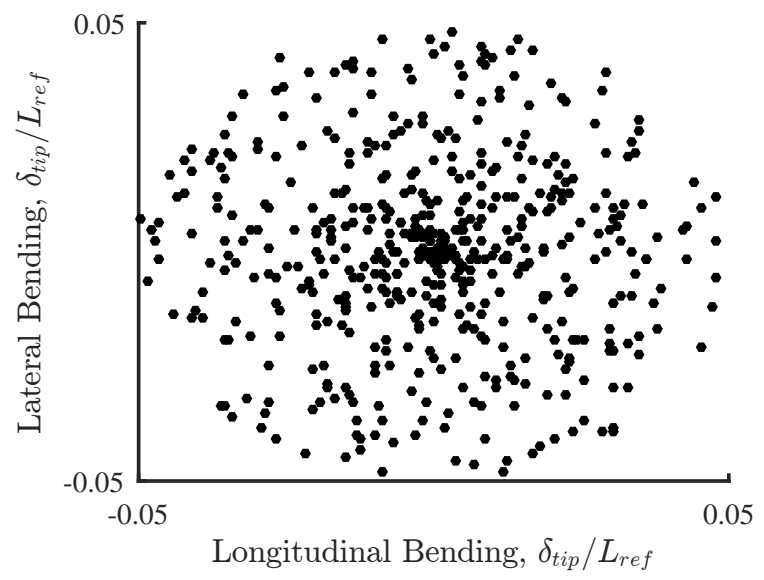

(c) Structural Deformation

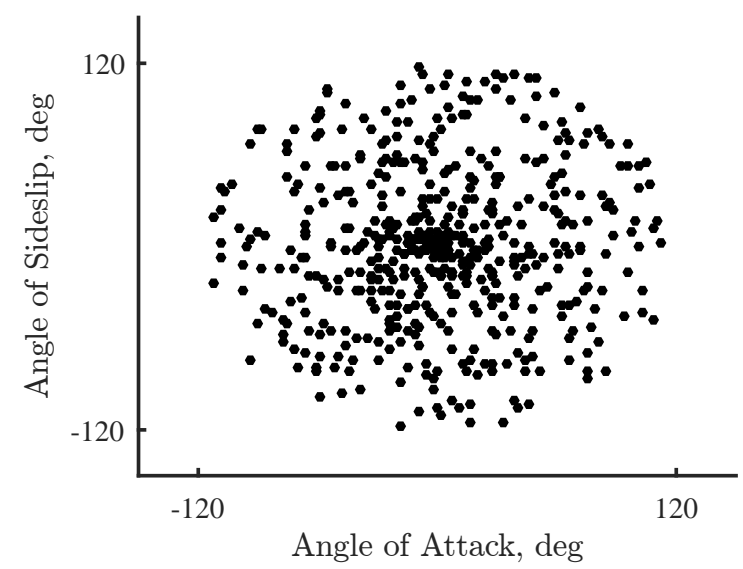

(b) Flow Angle of Incidence

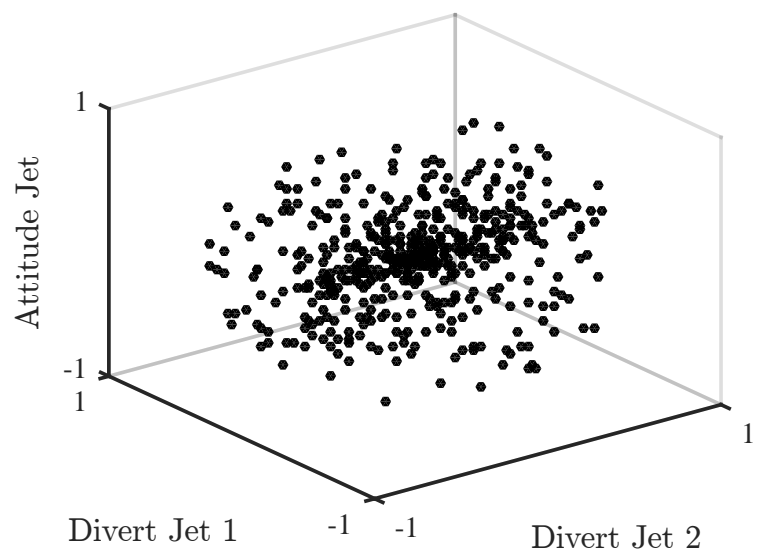

(d) Jet Throttle

Figure 3. Ranges of the steady-state CFD samples across 9 model input dimensions of the flow, structure, and jet parameters across the flight envelope

change in outputs is recorded. The output of the system is the vehicle loading in terms of the three bodyframe translation forces, three moments in the body frame and the generalized aerodynamic forces (GAFs) for each structural mode. During the simulation the unsteady loads $y$ are approximated using a linearized model. Therefore, the nonlinear loads $y_{\text {nonlinear }}^{u}$ calculated by using the nonlinear correction factor approach described in Section II.

Table 3. Input parameters for the unsteady FSJI surrogate model

\begin{tabular}{rll}
\hline \hline Parameter & Range & Units \\
Altitude & $12-30(40-100)$ & $\mathrm{km}(\mathrm{kft})$ \\
Mach & $2-4$ & \\
Angle of Attack & $0-120$ & degrees \\
Angle of Sideslip & $0-120$ & degrees \\
Total Structural Deformation & $0-5 \%$ & Normalized Tip Displacement \\
Jet Total Pressure & Off-10 & $\mathrm{MPa}$ \\
\hline
\end{tabular}

During the flight simulation loop the time history of each input, $u^{i}$, is convolved with the step response, $H_{i}^{j}$, to approximate the change in output, $y^{j}$. Finally, the vehicle loads at time index $n$ are evaluated as the 
linear combination of all the step response convolutions in addition to the original steady-state loads. The load $y^{j}[n]$ is expressed as,

$$
y^{j}[n]=y^{j}[0]+\sum_{i=1}^{m} \sum_{k=1}^{n} H_{i}^{j}[n-k]\left(u^{i}[k]-u^{i}[k-1]\right)
$$

where $m$ is the number of inputs. In the convolution approach the step responses are known at the sampled flight conditions, but must be approximated as the vehicle maneuvers and the conditions change. Therefore, a kriging model for how the step responses are varying is created that uses the flight conditions (altitude, Mach) to approximate the step responses $\hat{H}_{i}^{j}$.

\section{Results}

This section presents the verification of each of the modeling methods. The quality of the steady-state surrogate model and each of the linear unsteady models is assessed using a cross-validation approach. The combined unsteady nonlinear approximation is compared against a CFD simulation with varying flight conditions.

The leave-one-out approach is used as the cross-validation technique, which recursively removes one of the training samples to create a new surrogate model. The new model is then evaluated at the removed training point and the known removed solution is used as the reference to measure the level of error. This approach is especially useful for models with limited or sparse training data.

The first CFD verification maneuver is a forced motion simulation of the vehicle over a range of flight conditions. The vehicle orientation is prescribed at each time-step to simultaneously vary the angle of attack with a time-varying frequency. No structural deformation is prescribed during the simulation due to limitations on the code.

A second CFD verification maneuver that includes structural deformation along with the jet interaction component is presented. The structural deformation is prescribed as a sinusoidal signal with constant frequency and increasing amplitude at a un-sampled flight condition. This simulation tests the capability of the nonlinear correction factor approach in addition to the quality of the steady and unsteady surrogate models.

\section{A. Steady-State Model Verification}

The steady-state model is used to approximate the applied loads to the high-speed vehicle due to changes in the flight conditions in addition to the flow, structure, and jet parameters. Specifically, this model calculates the approximate surface pressure applied to the vehicle surface, which can then be integrated to calculate the six rigid-body forces and moments and the generalized loads for each structural deformation mode. The leave-one-out method is used to measure the quality of the surrogate steady-state model using the median absolute error (MAE) in the pressure normalized by the freestream dynamic pressure as well as the MAE of the integrated forces. The relative errors in the integrated forces, moments, and generalized forces are recorded for each leave-one-out evaluation. The statistics of these errors are described in terms of the $95 \%$ confidence interval of the median for each error metric and listed in Table 4. A sample from the crossvalidation of the surface pressure surrogate model is presented in Figure 4 with the conditions listed in Table 5 .

Table 4. Steady-State Model Cross-Validation Statistics

\begin{tabular}{cccccccc}
\hline \hline & $p / q_{\text {ref }}$ & $C_{x}$ & $C_{y}$ & $C_{z}$ & $C_{m x}$ & $C_{m y}$ & $C_{m z}$ \\
\hline Median MAE & 0.051 & 0.044 & 0.027 & 0.065 & 0.506 & 0.107 & 0.055 \\
95\% Confidence Lower Bound & 0.049 & 0.039 & 0.022 & 0.057 & 0.408 & 0.088 & 0.047 \\
95\% Confidence Upper Bound & 0.054 & 0.050 & 0.034 & 0.074 & 0.606 & 0.128 & 0.065 \\
\hline
\end{tabular}


Model

\section{CFD}

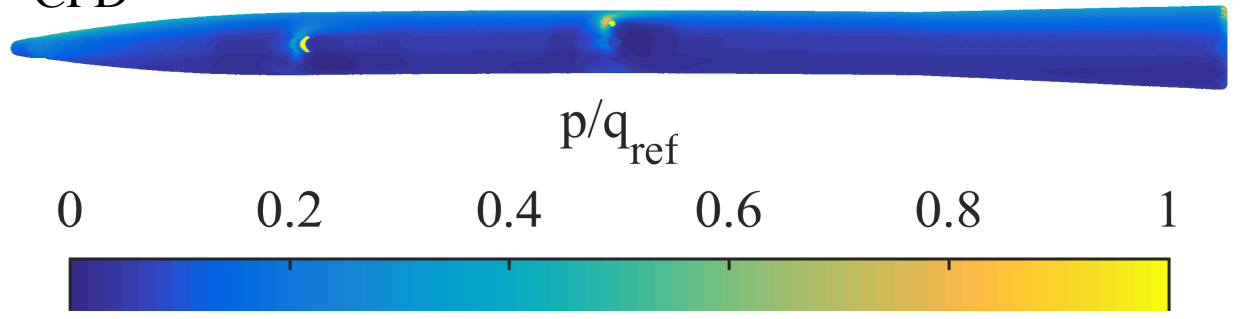

Figure 4. Pressure distribution of the model and CFD solution from one sample cross-validation point

Table 5. Sample cross-validation conditions of Figure 4

\begin{tabular}{ccl}
\hline \hline Parameter & Value & Unit \\
\hline Altitude & 19.5 & $\mathrm{~km}$ \\
Mach & 3.87 & \\
AoA & -8.88 & $\mathrm{deg}$ \\
AoS & -21.8 & $\mathrm{deg}$ \\
$\eta_{1}$ & 0.593 & \% Tip Displacement \\
$\eta_{2}$ & -1.12 & \% Tip Displacement \\
$\delta_{1}$ & 21.9 & \% Maximum \\
$\delta_{2}$ & -24.0 & \% Maximum \\
$\delta_{3}$ & 32.0 & \% Maximum \\
\hline
\end{tabular}

\section{B. Linear Unsteady Model Verification}

The unsteady model is based on the step response of the vehicle to changes in each input. However, these step responses are identified at select flight conditions, which then need to be interpolated to approximate the step response at un-sampled flight conditions during flight simulation. The output is the full step response at each time step and can be used directly with the time-history of the vehicle conditions to approximate the total unsteady loads using superposition. The collection of step responses collected from step changes in the angle of attack, tip displacement, and jet total pressure are presented along the left column in Figure 5. The $L_{2}-$ norm relative error obtained for each flight condition is calculated against the known solution for each modeling approach and the $95 \%$ confidence interval on the median error is presented in Table 6. Samples from the cross-validation are presented along the right column of Figure 5 that compare the predicted step response to the training set.

Table 6. Linear Unsteady Model Cross-Validation Statistics

\begin{tabular}{cccc}
\hline \hline & $\Delta A o A$ & $\Delta \eta_{1}$ & $\Delta \delta_{1}$ \\
$L_{2}$ relative error & 0.039 & 0.022 & 0.679 \\
$95 \%$ Confidence Lower Bound & 0.018 & 0.015 & 0.434 \\
$95 \%$ Confidence Upper Bound & 0.064 & 0.086 & 0.938 \\
\hline
\end{tabular}




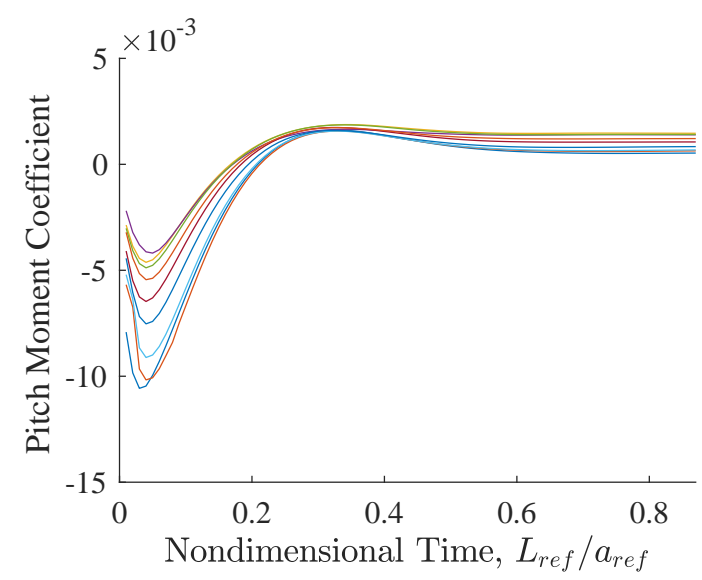

(a) Training Set

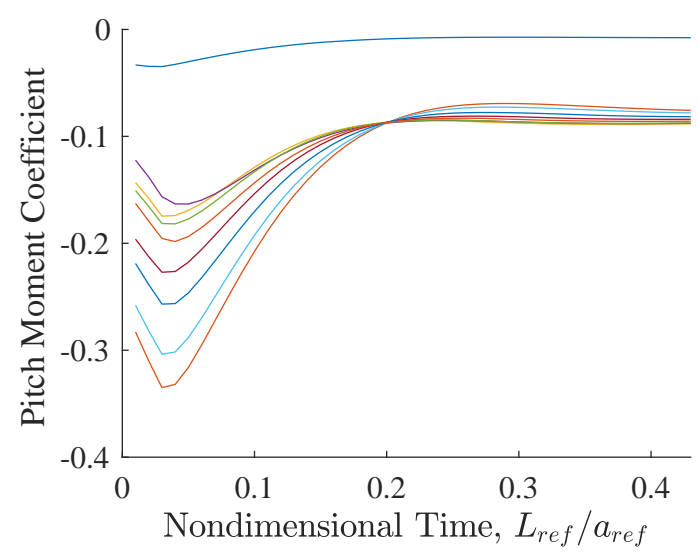

(c) Training Set

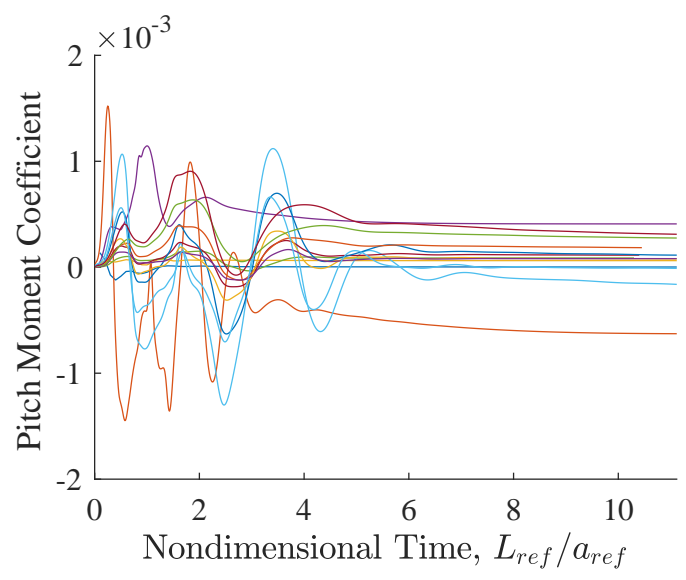

(e) Training Set

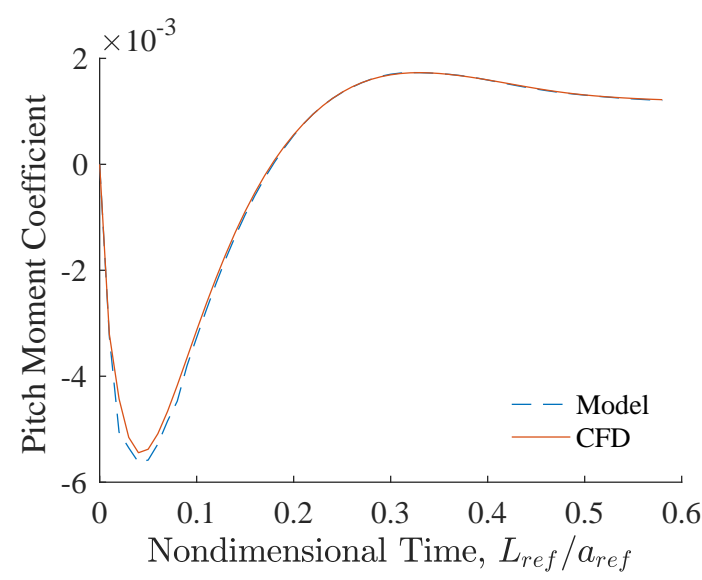

(b) Cross-Validation Sample

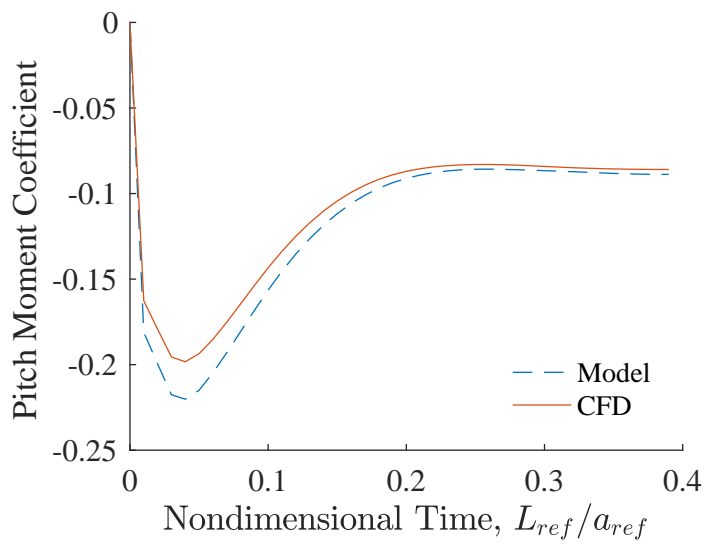

(d) Cross-Validation Sample

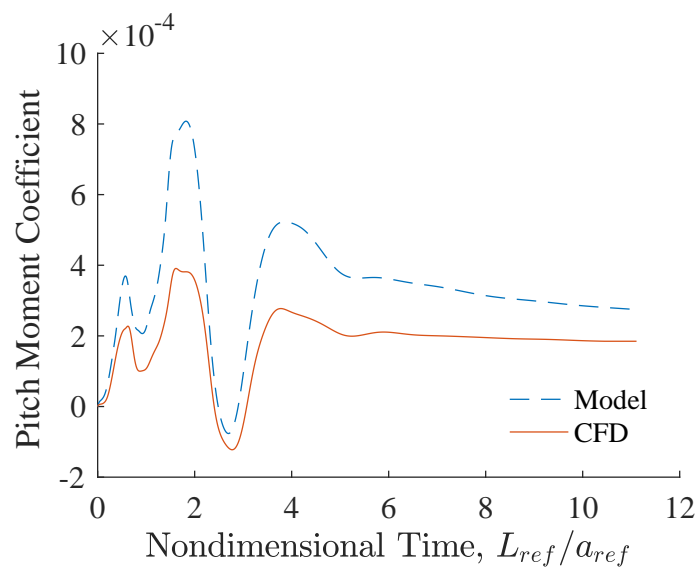

(f) Cross-Validation Sample

Figure 5. Step Responses to changes in $A o A, \eta_{1}$, and $\delta_{1}$ along with samples from the cross-validation of the training set

\section{Unsteady Nonlinear Model Verification}

The unsteady nonlinear FSJI is approximated using a combination of one of the linear unsteady models and the nonlinear steady-state model. The quality of each surrogate model is been presented, but the function of interest is the solution from time-accurate CFD simulation of varying flow, structure, and jet conditions. 
Three verification maneuvers were chosen to evaluate the nonlinear correction factor approach in addition to ability to capture unsteady FSJI effects. Similar to the steady-state model verification results, the relative errors in the integrated forces and moments are presented in Figure 6.

The first verification maneuver is the prescribed motion of an undeformed vehicle with varying flight conditions. The Reynolds number, deformation, and jet conditions relative to the farfield conditions are kept constant and listed in Table 7. The rotation is then prescribed at each time step to follow a sinusoidal maneuver with a moderate amplitude and increasing Mach number as a function of time. The second verification maneuver is the prescribed structural deformation of the vehicle as a function of time at constant flow and jet conditions listed in Table 7. The third maneuver is the response of a flexible vehicle to a step change in the jet total pressure of $1 \%$ of the maximum available total pressure and flight conditions listed in Table 8. The vehicle response is then approximated using each of the modeling methods and presented alongside the CFD time-accurate solution for each maneuver.

Table 7. Dynamic Rigid-Body and Structural Deformation Simulation Conditions

\begin{tabular}{ccc}
\hline \hline Altitude, km & Mach & Jet Total Pressure \\
21.3 & 3.0 & $17.5 \mathrm{MPa}$ \\
\hline
\end{tabular}

Table 8. Dynamic Jet Step Simulation Conditions

\begin{tabular}{cccc}
\hline \hline Altitude, $\mathrm{km}$ & Mach & Jet Step $(t=0)$ & Jet Step Total Pressure $(t>0), \mathrm{kPa}$ \\
21.3 & 3.0 & Off & 100 \\
\hline
\end{tabular}

For each simulation the flight conditions are used to approximate the step response of the system to changes in the fluid, structure, and jet conditions. These responses are then convolved with the input time history of each simulation to calculate the linearized model response. In the case of the final simulation with a jet step and flexible vehicle response these linearized outputs are summed together to reach the total linearized model response. Then, the nonlinear correction factor is calculated for all time steps by evaluating the steady-state surrogate model (Steady in Figure 6) and linearized model steady-state values. This correction factor is applied to the linearized model output and is referred to as the Unsteady Model in Figure 6.

The results in Figure 6 show that the steady and unsteady models do an adequate job of approximating the FSJI solution to rigid-body and structural deformation inputs. In addition, the unsteady model augments the steady-state solution further in the structural deformation case to better match the amplitude increase that is observed in the CFD solution. At worst, the the unsteady model matches the steady-state prediction and does not degrade the solution. The transient jet interaction solution of the third simulation is misidentified by the surrogate which leads to an incorrect transient solution and eventual phase difference in the signal. This is most likely due to the insufficient sampling of the jet step response and larger relative errors. Despite this error the strategy overall captures the frequency and amplitude growth of the vibrating vehicle in the presence of jet interaction. The unstable response that is observed is not indicative of the true vehicle response because the thrust of the jet itself is not accounted for due to the replacement of the nozzle exit with the inflow boundary condition. Therefore, the jet interaction with the flow is the driver that leads to an instability that amplifies the generalized force on the structure similar to the results in the jet sensitivity study.

The differences between the unsteady and steady model come from the unsteady component being excited and having an additional contribution, which is seen in the structural deformation case (Figure 6(c)-(d)). The differences between the unsteady model and the CFD solution are additional effects that are not fully captured using the linear convolution approach. This effects may include the nonlinearity of the jet interaction at higher jet total pressure, the sensitivity of the jet interaction effects to unsteadiness in the flow, and nonlinearity introduced by the combination of unsteadiness and stronger interactions between the jet and fluid. These effects may be addressed using a nonlinear representation of the system using a Volterra series and other approaches. 


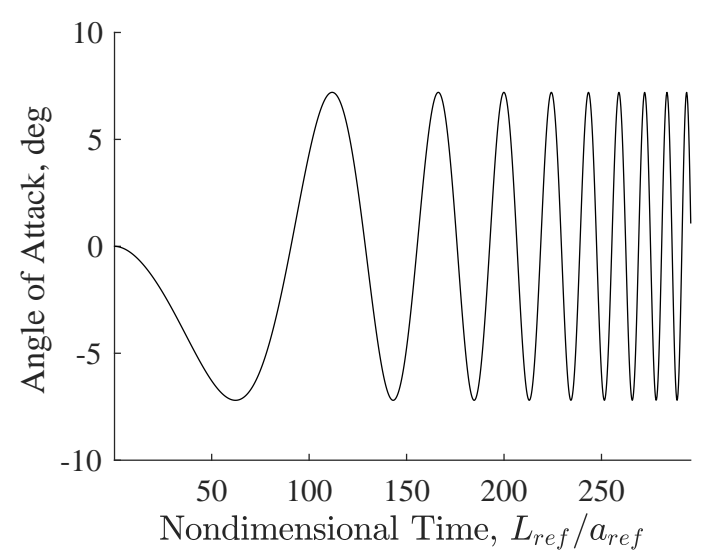

(a) Rigid-Body Input

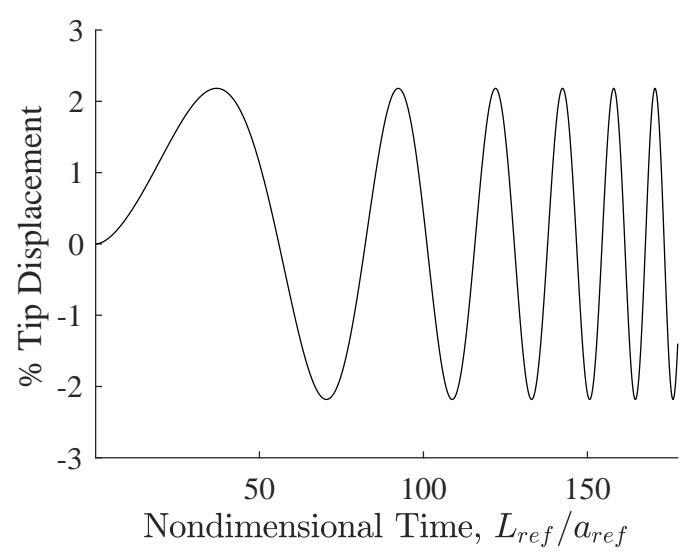

(c) Structural Deformation Input

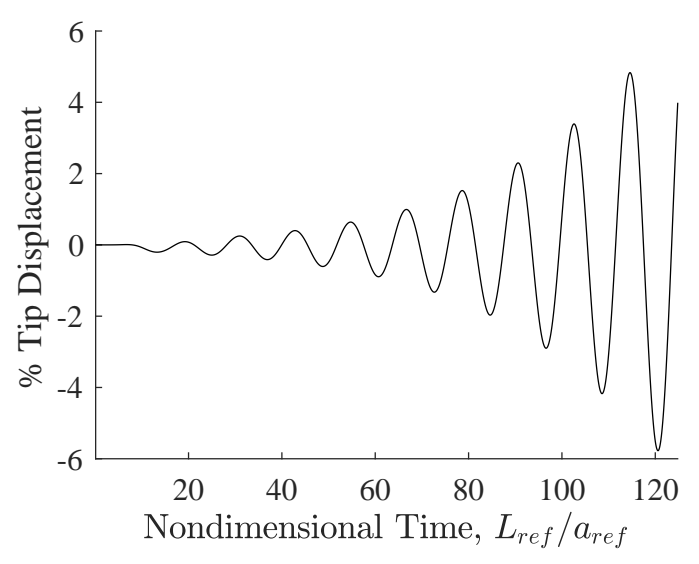

(e) Structural Deformation Response to Jet Step

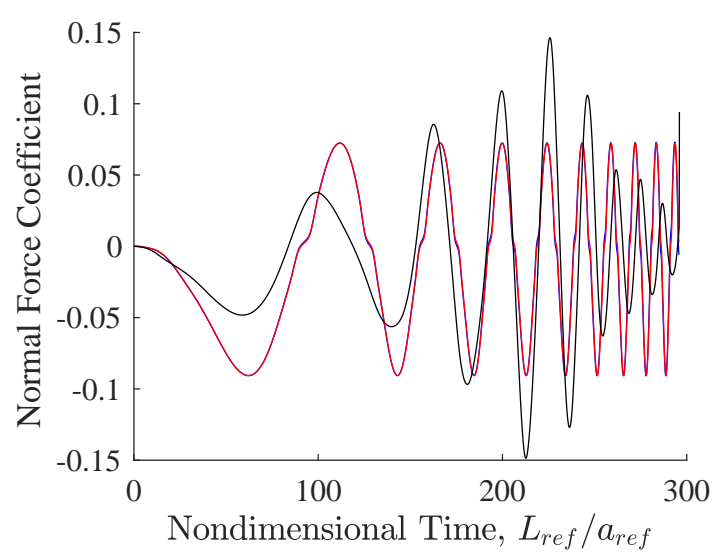

(b) CFD Solution and Model Output

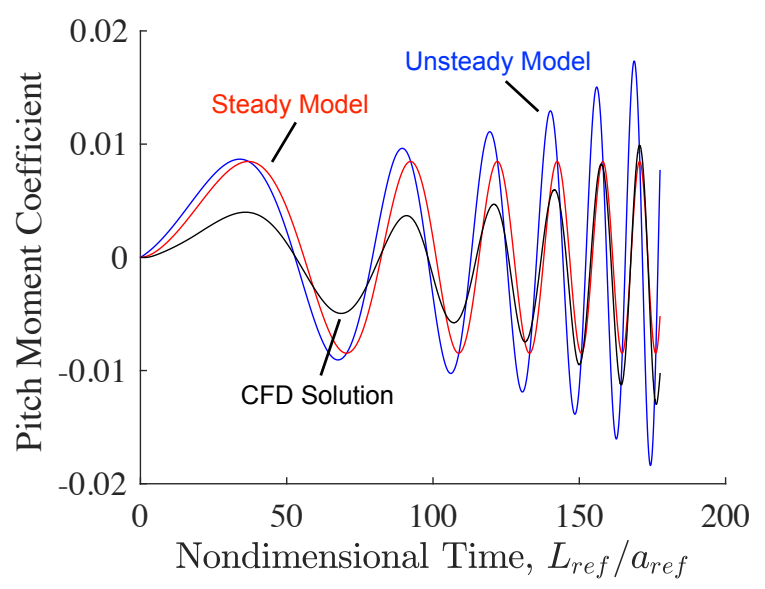

(d) CFD Solution and Model Output

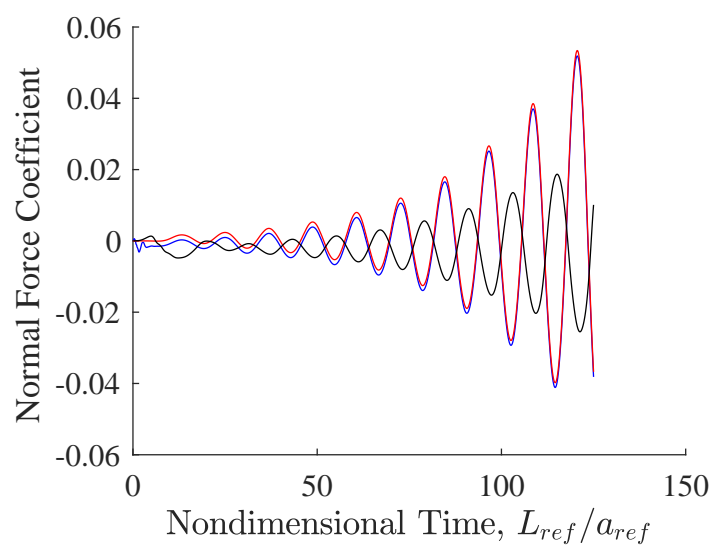

(f) CFD Solution and Model Output

Figure 6. Comparisons to CFD simulations of force pitch oscillation, forced structural deformation, and elastic response to transient jet actuation at the mean flight condition of $21.34 \mathrm{~km}$ at Mach 3.0. CFD solution, Steady-State Model, Unsteady Model

\section{Concluding Remarks}

This paper considers the unsteady fluid-structure-jet interaction that occurs for highly maneuverable, agile, high-speed vehicles. The coupled FSJI has a significant effect on the applied loads to the vehicle that 
must be modeled accurately to calculate the vehicle response. Flight simulation of the vehicle is desired to experiment with varying vehicle designs and aid the design and development process. The unsteady FSJI model must fit within a flight simulation framework, which requires an accurate, robust, and fast approach. A couple of methods that have been used for other unsteady aerospace applications are adapted for the unsteady FSJI problem.

One major challenge to modeling the unsteady loads applied to the maneuverable high-speed vehicle is the wide range of a high dimensional input problem. Developing a model for a limited number of flight conditions is sufficient for conventional air vehicles, but the whole flight envelope must be represented for a maneuverable vehicle with an undetermined trajectory. This adds more dimensions to the problem that is dependent on the fluid, structure, and jet states. The fluid, structure, and jet input parameters have nonlinear effects on the applied loads which adds an additional layer of complexity to the model. To address this wide range of input parameters over the entire flight envelope, the steady-state response is sparsely sampled before doing any unsteady sampling.

The two methods used in this work are the steady-state nonlinear surrogate model and the linear convolution of step responses with nonlinear correction. This approach perturbs each of the flow, structure, and jet conditions at a specified point within the flight envelope of altitude and Mach number to construct a linearized model. The linearized model accross several points in the flight envelope is then interpolated to create an approximate linearized model at unsampled flight conditions.

The modeling approach within the flight simulation framework takes the flight conditions to approximate the linearized unsteady model. Then the time history of the vehicle in terms of attitude, deformation, control inputs is used with the linearized unsteady model to calculate the linearized prediction of the unsteady vehicle loads. The current vehicle state is then used with the steady-state approximation model to calculate the appropriate nonlinear correction factor. In total this approach calls one unsteady model and one steadystate model to approximate the nonlinear unsteady loads of the high-speed vehicle performing aggressive maneuvers at unsampled conditions throughout the flight envelope.

The modeling approach involves two layers of surrogate models to create a comprehensive surrogate model of the nonlinear unsteady applied loads. Verification of each modeling layer and the composite model is required to measure the level of accuracy relative to the true solution. In each case the high-dimensionality of the problem imposes a significant cost for additional sampling for verification. Therefore, a cross-validation technique is used to measure the accuracy of each of the modeling sub-layers: the nonlinear steady-state model and linearized unsteady model. The full nonlinear unsteady model is compared to a select set of dynamic maneuvers meant to excite the unsteady FSJI effects on the vehicle applied loading.

The jet interaction effect appears to be sensitive to unsteadiness in the flow such that the steady-state model does not capture low-frequency inputs that well. The unsteady model based on the step responses of the vehicle contributes some additional information that is not represented in the steady model such as the transient jet interaction in response to turning the jet on and the amplification of the applied forces due to dynamic structural deformation. However, the limited number of samples for the jet step response led to an misrepresentation of the transient jet interaction. In addition, the unsteady model is still significantly off from the CFD solution. The gap between the current unsteady modeling approach and the CFD solution may be addressed using a nonlinear representation as the unsteady model is unable to capture even moderate flow unsteadiness for strong jet interactions.

Overall, this work adds to the discussion of unsteady loads prediction of highly nonlinear, high-dimensional, strongly coupled problems using a maneuverable high-speed vehicle as an example. A couple of modeling approaches from the literature are investigated to study the feasibility of using these approaches for this application. The modeling methods and approach developed is general and can be adapted for future use with multi-disciplinary problems. The nonlinear unsteady surrogate model of the FSJI for high-speed vehicles in particular enables flight simulation and aids the future design and development of high-performance vehicles.

\section{Acknowledgments}

This work was supported by the U.S. Air Force Research Laboratory Munitions Directorate with Dr. Crystal Pasiliao and Dr. Daniel Reasor as technical monitors. The CFD results were obtained primarily using the Lightning high performance computing system of the U.S. Air Force Research Laboratory DoD Supercomputing Resource Center. Opinions, interpretations, conclusions, and recommendations are those 
of the authors and are not necessarily endorsed by the U.S. Government.

\section{References}

${ }^{1}$ Spaid, F. W. and Zukoski, E. E., "A Study of the Interaction of Gaseous Jets from Transverse Slots with Supersonic

External Flows," AIAA Journal, Vol. 6, No. 2, February 1968, pp. 205-212.

${ }^{2}$ Spaid, F. W. and Cassel, L. A., "Aerodynamic Interference Induced by Reaction Controls," Tech. Rep. 173, AGARD, December 1973.

${ }^{3}$ Zukoski, E. E. and Spaid, F. W., "Secondary Injection of Gases into a Supersonic Flow," AIAA Journal, Vol. 2, No. 10, 1964, pp. 1689-1696.

${ }^{4}$ Roger, R. P., "The Aerodynamics of Jet Thruster Control for Supersonic/Hypersonic Endo-Intercepters: Lessons Learned," 37th AIAA Aerospace Sciences Meeting and Exhibit, AIAA 99-0804, January 1999.

${ }^{5}$ Billig, F. S., Orth, R. C., and Lasky, M., "A Unified Analysis of Gaseous Jet Penetration," AIAA Journal, Vol. 9, No. 6, June 1971, pp. 1048-1058.

${ }^{6}$ Werle, M. J., Driftmyer, R. T., and Shaffer, D. G., "Jet-Interaction-Induced Separation: The Two-Dimensional Problem," AIAA Journal, Vol. 10, No. 2, February 1972, pp. 188-193.

7 ${ }^{7}$ Young, C. T. K. and Barfield, B. F., "Viscous Interaction of Sonic Transverse Jets with Supersonic External Flows," AIAA Journal, Vol. 10, No. 7, July 1972, pp. 853-854.

${ }^{8}$ Demuren, A. O., "Modeling Jets in Cross Flow," Tech. Rep. NASA-CR-194965, NASA, 1994.

$\checkmark{ }^{9}$ Broadwell, J. E., "Analysis of the Fluid Mechanics of Secondary Injection for Thrust Vector Control," AIAA Journal, Vol. 1, No. 5, May 1963, pp. 1067-1075.

$\checkmark{ }^{10}$ Warfield, M. J., "Calculation of Supersonic Interacting Jet Flows," 27th Aerospace Sciences Meeting, AIAA 89-0666, January 1989.

${ }^{11}$ Graham, M. J. and Weinacht, P., "Numerical Simulation of Lateral Control Jets," 37th AIAA Aerospace Sciences Meeting and Exhibit, AIAA 99-0510, January 1999.

${ }^{12}$ Sahu, J., Fresconi, F., and Heavey, K. R., "Unsteady Aerodynamic Simulations of a Finned Projectile at a Supersonic Speed With Jet Interaction," Tech. Rep. ARL-TR-6960, U.S. Army Research Laboratory, Aberdeen Proving Ground, MD, June 2014.

${ }^{13}$ DeSpirito, J., "Turbulece Model Effects on Cold-Gas Lateral Jet Interaction in a Supersonic Crossflow," Journal of Spacecraft and Rockets, Vol. 52, No. 3, May-June 2015, pp. 836-852.

${ }^{14}$ DeSpirito, J., Kennedy, K. D., Mikkelsen, C. D., and Chaplin, R. A., "Predictions of a Supersonic Jet-in-Crossflow: Comparisons Among CFD Solvers and with Experiment," 32nd AIAA Applied Aerodynamics Conference, AIAA 2014-2736, June 2014.

$\checkmark{ }^{15}$ DeSpirito, J., "Lateral Jet Interaction on a Finned Projectile in Supersonic Flow," 50th AIAA Aerospace Science Meeting including the New Horizons Forum and Aerospace Exposition, AIAA 2012-0413, January 2012.

-16DeSpirito, J., "Factors Affecting Reaction Jet Interaction Effects on Projectiles," 29th AIAA Applied Aerodynamics Conference, AIAA 2011-3031, June 2011.

-17DeSpirito, J., "Transient Lateral Jet Interaction Effects on a Generic Fin-Stabilized Projectile," 30th AIAA Applied Aerodynamics Conference, AIAA 2012-2907, June 2012.

$\checkmark{ }^{18}$ Gnemmi, P., Adeli, R., and Longo, J., "Computational Comparisons of the Interaction of Lateral Jet on a Supersonic Generic Missile," AIAA Atmospheric Flight Mechanics Conference and Exhibit, No. AIAA 2008-6883, 2008.

19 Ebrahimi, H. B., "Numerical Simulation of Transient Jet-Interaction Phenomenology in a Supersonic Freestream," Journal of Spacecraft and Rockets, Vol. 37, No. 6, November-December 2000, pp. 713-719.

${ }^{20}$ Naumann, K. W., Ende, H., George, A., and Mathieu, G., "Stationary and Time-Dependent Effects in the Near Interaction of Gaseous Jets and Supersonic Cross-flow," 29th AIAA Fluid Dynamics Conference, AIAA 1998-2972, 1998.

$\checkmark{ }^{21}$ Ferrante, A., Matheou, G., and Dimotakis, P. E., "LES of an Inclined Jet into a Supersonic Turbulent Crossflow: Synthetic Inflow Conditions," 48th AIAA Aerospace Sciences Meeting Including the New Horizons Forum and Aerospace Exposition, AIAA 2010-1287, January 2010.

${ }^{22}$ Kawai, S. and Lele, S. K., "Large-Eddy Simulation of Jet Mixing in Supersonic Crossflows," AIAA Journal, Vol. 48, No. 9, 2010, pp. 2063-2083.

${ }^{23}$ VanderWyst, A. S., Shelton, A. B., Martin, C. L., Neergaard, L. J., and Witeof, Z. D., "Reduced Order Models for Generation of Large, High Speed Aerodynamic Databases with Jet Interactions," 57th AIAA/ASCE/AHS/ASC Structures, Structural Dynamics and Materials Conference, AIAA 2016-0464, January 2016.

${ }^{24}$ VanderWyst, A., Shelton, A., and Martin, C., "A Computationally Efficient, Multi-fidelity Assessment of Jet Interactions for Highly Maneuverable Missiles," 34th AIAA Applied Aerodynamics Conference, No. AIAA 2016-4333, 2016.

${ }^{25}$ Raveh, D. E. and Mavris, D. N., "Reduced-Order Models Based on CFD Impulse and Step Responses," 42nd AIAA/ASME/ASCE/AHS/ASC Structures, Structural Dynamics, and Materials Conference, AIAA 01-1527, April 2001.

${ }^{26}$ Skujins, T. and Cesnik, C. E. S., "Reduced-Order Modeling of Unsteady Aerodynamics Acorss Multiple Mach Regimes," Journal of Aircraft, Vol. 51, No. 6, November-December 2014, pp. 1681-1704.

${ }^{27}$ Zaide, A. and Raveh, D. E., "Numerical Simulation and Reduced-Order Modeling of Airfoil Gust Response," AIAA Journal, Vol. 44, No. 8, 2006, pp. 1826-1834.

${ }^{28}$ Biedron, R. T., Carlson, J., Derlaga, J. M., Gnoffo, P. A., Hammond, D. P., Jones, W. T., Kleb, B., Lee-Rausch, E. M., Nielsen, E. J., Park, M. A., Rumsey, C. L., Thomas, J. L., and Wood, W. A., "FUN3D Manual 12.9," Tech. Rep. NASA-TM-2016-219012, NASA, 2016.

${ }^{29}$ Kitson, R. C. and Cesnik, C. E. S., "Aeroelastic Modeling and Simulation of High-Speed Flexible Vehicles," 15th Dynamics Specialists Conference, AIAA 2016-1324, January 2016. 